\title{
Coherent coupling of individual quantum dots measured with phase-referenced two-dimensional spectroscopy: Photon echo versus double quantum coherence
}

\author{
Valentin Delmonte, ${ }^{1,2}$ Judith F. Specht,${ }^{3}$ Tomasz Jakubczyk, ${ }^{1,2}$ Sven Höfling,,${ }^{4,5}$ Martin Kamp, ${ }^{4}$ Christian Schneider ${ }^{4}$ \\ Wolfgang Langbein, ${ }^{6}$ Gilles Nogues, ${ }^{1,2}$ Marten Richter, ${ }^{3}$ and Jacek Kasprzak ${ }^{1,2, *}$ \\ ${ }^{1}$ Université Grenoble Alpes, F-38000 Grenoble, France \\ ${ }^{2}$ CNRS, Institut Néel, "Nanophysique et Semiconducteurs" Group, F-38000 Grenoble, France \\ ${ }^{3}$ Institut für Theoretische Physik, Nichtlineare Optik und Quantenelektronik, Technische Universität Berlin, \\ Hardenbergstrasse 36, D-10623 Berlin, Germany \\ ${ }^{4}$ Technische Physik, University of Würzburg, Würzburg 97074, Germany \\ ${ }^{5}$ SUPA, School of Physics and Astronomy, University of St. Andrews, St. Andrews KY16 9SS, United Kingdom \\ ${ }^{6}$ School of Physics and Astronomy, Cardiff University, The Parade, Cardiff CF24 3AA, United Kingdom
}

(Received 9 March 2017; published 26 July 2017)

\begin{abstract}
We employ two-dimensional (2D) coherent, nonlinear spectroscopy to investigate couplings within individual InAs quantum dots (QDs) and QD molecules. Swapping pulse ordering in a two-beam sequence permits one to distinguish between rephasing and nonrephasing four-wave mixing (FWM) configurations. We emphasize the nonrephasing case, allowing one to monitor two-photon coherence dynamics. Respective Fourier transform yields a double quantum 2D FWM map, which is corroborated with its single quantum counterpart, originating from the rephasing sequence. We introduce referencing of the FWM phase with the one carried by the driving pulses, overcoming the necessity of its active stabilization, as required in 2D spectroscopy. Combining single and double quantum 2D FWM provides a pertinent tool in detecting and ascertaining coherent coupling mechanisms between individual quantum systems, as exemplified experimentally.
\end{abstract}

DOI: 10.1103/PhysRevB.96.041124

Nuclear magnetic resonance (NMR) spectroscopy conceived phase-locked, multipulse techniques, yielding multidimensional spectra by Fourier transforming temporal sequences into respective frequency coordinates $[1,2]$. The possibility to spread the response of biological or chemical molecules of high structural complexity, especially proteins, across many axes enabled one to assess their spatial form and to understand interatomic interactions and couplings. The idea to selectively address and evolve subsets of transitions from congested spectra via a multipulse toolbox, and then projecting the results onto two-dimensional (2D) or three-dimensional diagrams, is a far-reaching legacy of NMR. At a juncture of coherent spectroscopy and condensed matter physics, 2D spectroscopy provided insight into the dynamics and couplings of many-body optical excitations in solids, in particular, of excitons in semiconductor quantum wells [3-5] and novel 2D layered materials [6], as well as in ensembles of quantum dots [7,8] (QDs) or nanocrystals [9]. A principal tool in these investigations is $k$-resolved four-wave mixing (FWM) spectroscopy and its extensions probing multiwave mixing processes [4].

FWM spectroscopy has been exploited over the years to study Coulomb interactions and related ultrafast coherent dynamics of excitons in semiconductors [10-14]. In these ensemble experiments, a strong inhomogeneous broadening usually is an obstacle to implement coherent control protocols.

\footnotetext{
*jacek.kasprzak@neel.cnrs.fr
}

Published by the American Physical Society under the terms of the Creative Commons Attribution 4.0 International license. Further distribution of this work must maintain attribution to the author(s) and the published article's title, journal citation, and DOI.
This issue is largely overcome when restricting the study to individual excitons. Moreover, in an exciting context of optical information processing in solids, establishing controlled channels of coupling within a set of few-level systems represents a truly challenging goal. For these reasons, it is necessary to access the coherence of individual excitons, and then to ascertain the mechanism of their coherent interactions, involving both Coulomb (local) and radiation (long-range) mediated phenomena. The present Rapid Communication represents a step forward in this field.

FWM microscopy of single QD excitons [15] was previously accomplished by phase-sensitive optical heterodyning combined with interferometric detection, efficiently subtracting resonant background and permitting colinear geometry of the excitation pulses. Recently, the detection sensitivity of intrinsically weak single QD FWM has been enhanced substantially by using photonic nanostructures, improving the QD coupling with external laser beams [16-18]. Here, we perform FWM spectroscopy of individual InAs QDs embedded in a low- $Q$ semiconductor microcavity [19]. We point out two advancements with respect to our recent reports [16,20]: First, we demonstrate 2D FWM constructed from two-photon coherences-known as double quantum 2D FWM [21-24]-driven on individual transitions, specifically QD exciton-biexciton systems $(G X B)$ [25]. Second, we introduce referencing of the FWM phase, offering a convenient alternative for its active stabilization, which is widely believed to be required in 2D spectroscopy. Using the one-quantum and two-quantum spectroscopy, we have measured single QDs and a QD molecule. A comparison of the spectra signatures to theory allowed us to identify the nature of the internal coupling mechanism in the QD molecule system. Our work shows that the combined single and double quantum 2D spectroscopy is a powerful tool to reveal and understand coherent coupling 

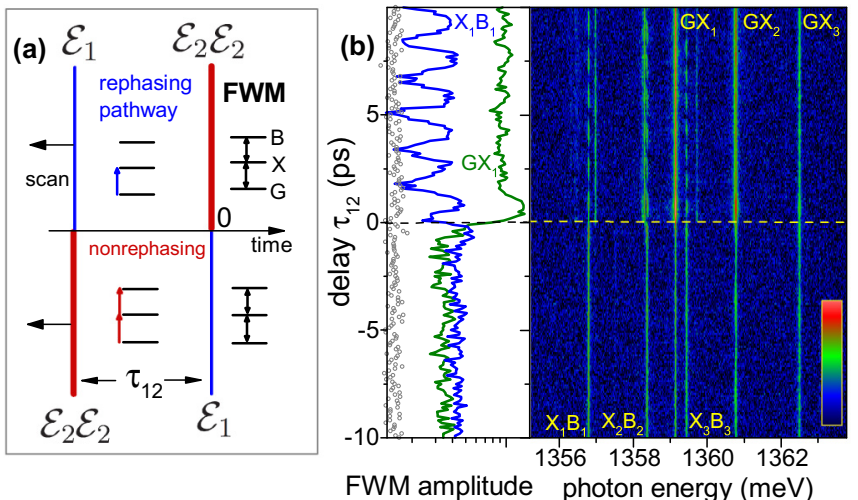

FIG. 1. Rephasing and nonrephasing pathways in two-beam four-wave mixing of individual quantum dots. (a) Two possible pulse sequences in the so-called positive (negative) delays $\tau_{12}$, corresponding to the rephasing (nonrephasing) FWM pathways. The nonrephasing pathway involves a two-photon coherence between the ground state $(G)$ and a two-particle state, here, a quantum dot biexciton $(B)$. (b) Measured FWM amplitude as a function of $\tau_{12}$ on a few InAs QDs embedded in a low- $Q$ microcavity. Impinging $\mathcal{E}_{1}, \mathcal{E}_{2}$ intensities of $(150,600) \mathrm{nW}$ correspond to pulse areas of around $(0.4 \pi, 0.8 \pi)$, significantly beyond the $\chi^{(3)}$ limit, generating a pronounced exciton-biexciton beating.

and excitation transfer mechanisms - an interdisciplinary issue spanning from biology and photochemistry, to quantum engineering. The results are especially pertinent for the latter area, as we open different avenues of research in the quantum control of optically active nanoscopic two-level and few-level systems in solids.

To acquire the FWM spectra [16], we use a pair of 100 fs laser pulses, $\mathcal{E}_{1}$ and $\mathcal{E}_{2}$, with a variable delay $\tau_{12}$, positive for $\mathcal{E}_{1}$ leading. They are frequency shifted by $\Omega_{1}=$ $80 \mathrm{MHz}$ and $\Omega_{2}=80.77 \mathrm{MHz}$, respectively, using acoustooptic deflectors. A FWM heterodyne beat with a reference field $\mathcal{E}_{\mathrm{R}}$ is retrieved at $2 \Omega_{2}-\Omega_{1}=81.54 \mathrm{MHz}$ frequency, carrying the lowest-order response $\mathcal{E}_{1}^{\star} \mathcal{E}_{2} \mathcal{E}_{2}$ (where $\star$ denotes complex conjugate) and also higher orders with the same phase evolution. The signal is spectrally dispersed using a spectrometer, detected with a CCD camera and retrieved in amplitude and phase by applying spectral interferometry. $\mathcal{E}_{\mathrm{R}}$ arrives a few picoseconds prior to $\mathcal{E}_{2}$, unless specified otherwise.

As shown in Fig. 1(a), in two-beam FWM, the first pulse $\mathcal{E}_{1}$ induces coherence, which evolves during $\tau_{12}$, to be then converted into FWM by the second pulse $\mathcal{E}_{2}$. The lowest electronic excitations of a neutral QD can be cast into three categories of states: a ground state $(G)$, single excitons $(X)$, and two-exciton states, known as biexcitons $(B) . G X$ transitions are addressed by one-photon coherence driven by $\mathcal{E}_{1}$, which is converted to FWM of $G X$ and $X B$ by a density grating $\mathcal{E}_{1}^{\star} \mathcal{E}_{2}$ on $G$ and $X[20,26]$. Inverting the temporal ordering of the two light pulses, a $G B$ transition can be inspected by a two-photon coherence induced by $\mathcal{E}_{2}$, transformed into FWM of both transitions at the arrival of $\mathcal{E}_{1}$ [20,26]. The simple three-level system of Fig. 1(a) illustrates the case of a neutral QD driven along one of its polarization axes. For a single two-level system, such as a QD trion, FWM can be only

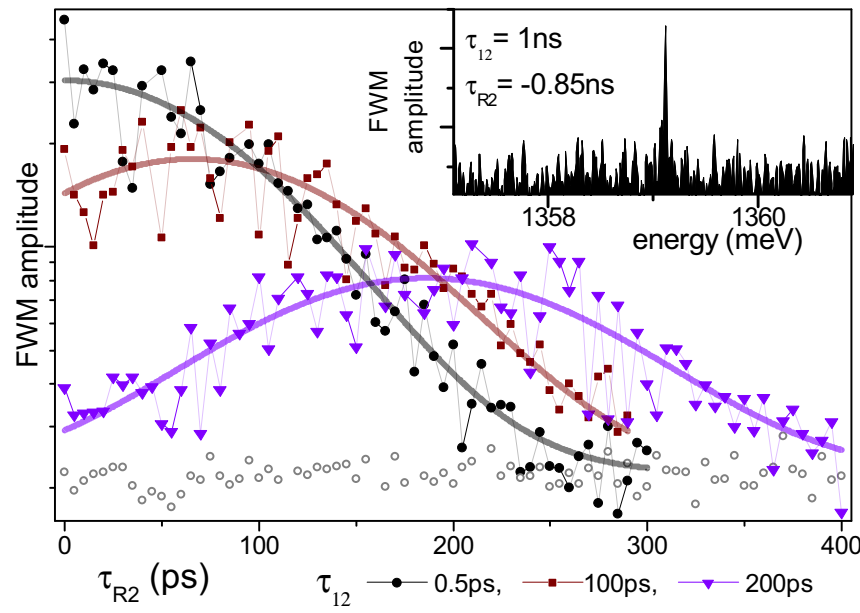

FIG. 2. Photon-echo formation on a single QD exciton measured upon a FWM rephasing pathway. The delay $\tau_{\mathrm{R} 2}$, between the reference $\mathcal{E}_{\mathrm{R}}$ and $\mathcal{E}_{2}$, is scanned for different values of $\tau_{12}$, as indicated. Formation of the photon echo is observed: A Gaussian form of the FWM transient is fully recovered for $\tau_{12}>\hbar / \sigma$. Temporal width of the echo yields the inhomogeneous broadening $\sigma$. Inset: By adjusting $\tau_{\mathrm{R} 2}$, one shifts the temporal detection window towards the echo, such that the FWM signal can be retrieved via spectral interference even for delays exceeding the temporal resolution of the setup, defined by the spectrometer. This is here exemplified for $\tau_{12}=1 \mathrm{~ns}$ and $\tau_{\mathrm{R} 2}=-0.85 \mathrm{~ns}$.

created for $\tau_{12}>0$ from one-photon coherence induced by $\mathcal{E}_{1}$, since the trion system cannot be doubly excited within the employed spectral bandwidth. In fact, two transitions in Fig. 1(b) show strictly no signal for $\tau_{12}<0$ and are attributed to trion transitions. Therein, we also recognize pairs of exciton biexcitons, labeled as $G X_{1}-X_{1} B_{1}, G X_{2}-X_{2} B_{2}$, and $G X_{3}-X_{3} B_{3}$, occurring in three distinct QDs. FWM exhibits a pronounced beating as a function of $\tau_{12}>0$, with a period corresponding to $B$ binding energy, which is induced beyond the $\chi^{(3)}$ regime by high-order contributions propagating at the FWM frequency [20,27]. Instead, for $\tau_{12}<0$, FWM is equally created on $G X$ and $X B$ transitions, with no beating.

A time-resolved FWM transient created upon the two pulse configurations displays different characteristics. For $\tau_{12}>0$, there is a phase conjugation between $\mathcal{E}_{1}$ and FWM. Owing to the rephasing, FWM of an inhomogeneously broadened system has a Gaussian form, with a maximum at $t=\tau_{12}$ and temporal width inversely proportional to the probed spectral inhomogeneous broadening $\sigma$. Importantly, the timeintegrated amplitude of such a photon echo is not sensitive on $\sigma$, instead, the homogeneous broadening is probed through the $\tau_{12}$ dependence. At a level of individual transitions, $\sigma$ is accumulated due to a residual spectral wandering in time-averaged measurements $[17,18,26,28]$. For $\sigma$ in the $\mu \mathrm{eV}$ range, which is a case even for high-quality QD systems, the echo width becomes comparable to or larger than the temporal sensitivity, given by the spectrometer resolution (here about $120 \mathrm{ps}$ ). To demonstrate the formation of such a broad echo [18], we scan the delay $\tau_{\mathrm{R} 2}$, between $\mathcal{E}_{\mathrm{R}}$ and $\mathcal{E}_{2}$, for three different $\tau_{12}$, as shown in Fig. 2 . The echo develops fully only for $\tau_{12}=200 \mathrm{ps}$, and from its width [full width at half maximum 

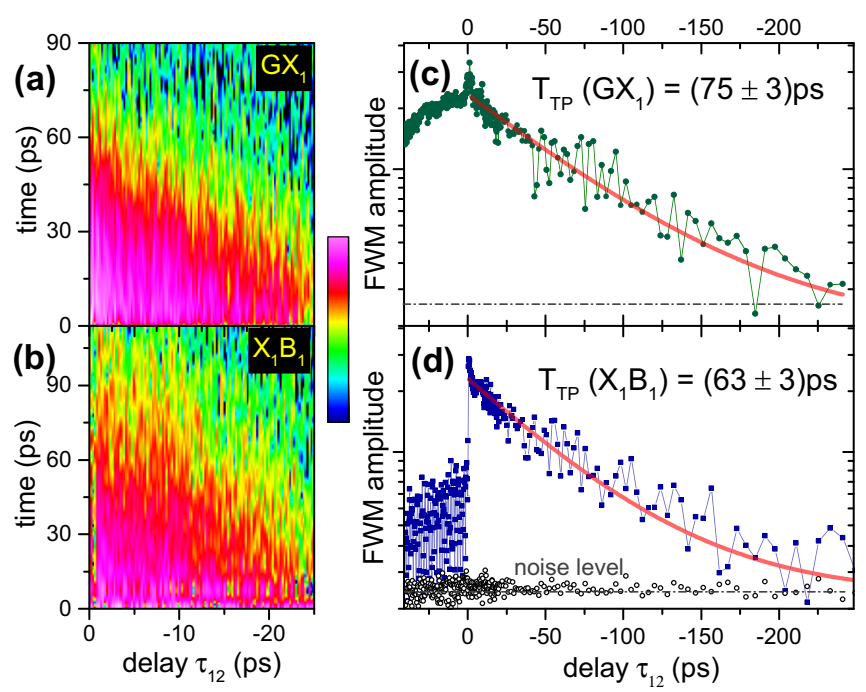

FIG. 3. Coherent dynamics of an exciton-biexciton system measured at the nonrephasing FWM configuration, $\tau_{12}<0$. (a), (b) Time-resolved FWM transient measured at $G X_{1}$ and $X_{1} B_{1}$ for negative delays. Due to the nonrephasing configuration, the FWM decay becomes more pronounced when increasing delay. (c), (d) Two-photon coherence dynamics, induced between $G$ and $B$ (also known as a biexciton coherence), measured at the nonrephasing FWM configuration. The two-photon dephasing time is retrieved from the exponential decay of $G X_{1}$ and $X_{1} B_{1}$ transitions.

(FWHM)] $t_{\sigma}=\hbar / \sigma=(214 \pm 33)$ ps we retrieve spectral inhomogeneous broadening $8 \ln (2) \sigma=8 \ln (2) \hbar / t_{\sigma}=(17 \pm$ 3) $\mu \mathrm{eV}$ (FWHM). By advancing $\tau_{\mathrm{R} 2}$ sufficiently close to the maximum of the echo, the temporal detection window is brought toward its maximum, permitting one to retrieve FWM spectral interference for delays significantly exceeding the temporal sensitivity of the spectrometer, as shown in the inset for $\tau_{12}=1 \mathrm{~ns}$ and $\tau_{\mathrm{R} 2}=-0.85 \mathrm{~ns}$.

For $\tau_{12}<0$ there is no strict phase conjugation between two-photon coherence and FWM, and therefore the photon echo is absent. In Figs. 3(a) and 3(b) we show $\left(t, \tau_{12}\right)$ resolved maps of the FWM amplitude measured on the $G X_{1}$ and $X_{1} B_{1}$ transitions, respectively. As $\tau_{12}$ is increased towards more negative values, FWM decay becomes more pronounced, owing to a nonrephasing character of the signal. The two-photon coherence dynamics of $G X_{1}$ and $X_{1} B_{1}$, i.e., respective time-integrated FWM vs $\tau_{12}$, are presented in Figs. 3(a) and 3(b). From the exponential decay of $G X_{1}$ and $X_{1} B_{1}$ we retrieve two-photon (biexciton) dephasing [29,30] $T_{\mathrm{TP}}\left(G X_{1}, X_{1} B_{1}\right)=(75 \pm 3,65 \pm 3) \mathrm{ps}$. Similar values of $T_{\mathrm{TP}}$ are obtained by analyzing two other $G X-X B$ pairs. Note that, in homogenously broadened $G X B$ systems, $T_{\mathrm{TP}}$ should be the same when inferring it either from the $G X$ or $X B$ transition. A slightly faster biexciton dephasing evaluated from the $X_{1} B_{1}$ decay is attributed to its stronger inhomogeneous broadening via spectral wandering with respect to $G X_{1}$ : The latter is due to energy fluctuations of the exciton level only, whereas the former is sensitive on wandering of both the exciton and biexciton levels [31,32]. These spectral fluctuations do not have to be correlated, and thus yield a shorter $T_{\mathrm{TP}}$ when reading it out from $X_{1} B_{1}$.
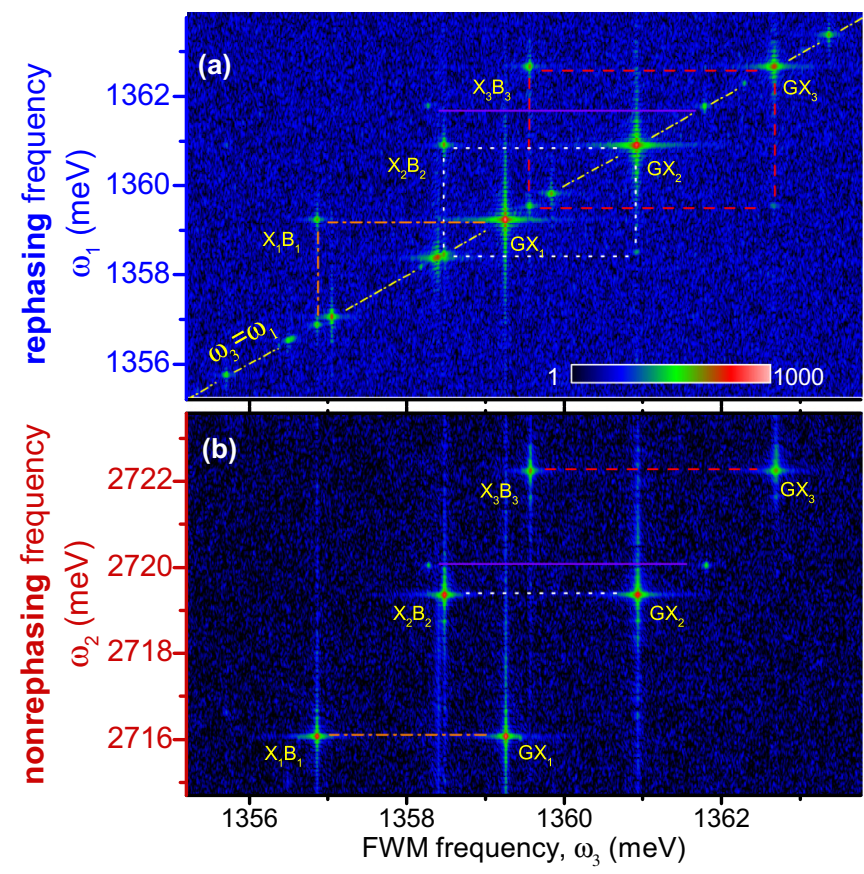

FIG. 4. Two-dimensional FWM spectroscopy of exciton complexes in a few InAs QDs probed along the (a) rephasing and (b) nonrephasing pathways. Four exciton-biexciton systems in different QDs are indicated dashed-dotted, dotted, dashed, and solid lines, respectively.

To illustrate couplings in the probed system of a few QDs, we Fourier transform $\operatorname{FWM}\left(\omega_{3}, \tau_{12}\right)$ sequences with respect to the delay $\tau_{12}$. The experimental setup is encapsulated, providing a passive stabilization of the phase during the acquisition. However, the phase relationship between FWM measured for subsequent $\tau_{12}$ is inevitably lost and can only be achieved via active stabilization $[33,34]$, which is not implemented here. Knowledge of the FWM phase for subsequent delays $\tau_{12}$ is a precondition to execute the Fourier transform yielding 2D FWM. In our previous works [20,35], we have circumvented this issue by imposing a phase relationship onto the data by choosing a separated transition in the spectral domain, acting as a local oscillator, and setting its phase to zero for all delays. We then applied this phase factor globally to the full spectrum, adjusting all other frequencies versus $\tau_{12}$, accordingly. Such a transformation remains justified, as long as the guiding transition to correct for, in particular, exhibiting no coherent coupling, is available in the spectrum. This generally is not the case. To overcome this experimental limitation, we have conceived a post-treatment protocol permitting one to reference the FWM phase, using auxiliary spectral interferences of $\mathcal{E}_{\mathrm{R}}$ with the driving pulses (see Sec. II A of the Supplemental Material [36] for a description of the phase-referencing protocol).

In Fig. 4 we present 2D FWM obtained from the set of QDs highlighted in Fig. 1. For $\tau_{12}>0$, FWM generated by all resonances driven by $\mathcal{E}_{1}$ forms a diagonal in the resulting 2D spectrum. This includes single trions and neutral excitons, but also biexcitons-the latter can directly be driven by $\mathcal{E}_{1}$ beyond the $\chi^{(3)}$ limit [20], as applied here (an example of the 2D FWM of $G X B$ systems in the $\chi^{(3)}$ limit is provided in 

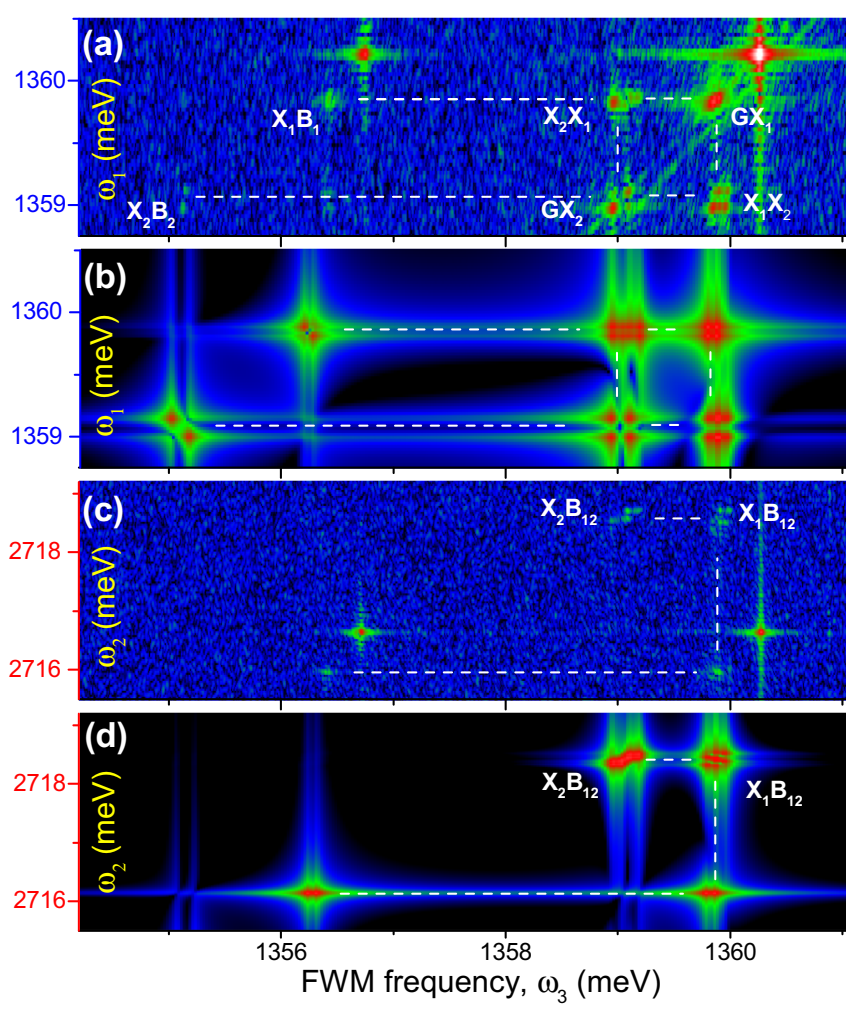

FIG. 5. Quantum dot molecule, consisting of two electrostatically coupled InAs QDs, observed in single and double quantum 2D FWM. Measured (a) rephasing and (c) nonrephasing 2D FWM spectra revealing coherent couplings between two QDs. Corresponding simulations (see Sec. I C of the Supplemental Material [36] for details regarding the model parameters) are shown in (b) and (d). The signatures belonging to this QD molecule are marked by dashed lines. An additional exciton-biexciton pair in (a) and (c) at $(1360.3,1356.8) \mathrm{meV}$ occurs in other QDs not involved in the molecule formation, thus not included in the calculated spectra.

Fig. S6 of the Supplemental Material [36]). The biexcitons are off-diagonally shifted by their respective binding energies of a few meV, and form squarelike features in 2D FWM under strong excitation, i.e., close to the $(\pi / 2, \pi)$ area of $\left(\mathcal{E}_{1}, \mathcal{E}_{2}\right)$ pulses. 2D FWM resulting from $\tau_{12}<0$ is shown in Fig. 4(b). FWM originates from a corresponding two-photon resonance driven by $\mathcal{E}_{2}$. Here, the two-photon energy corresponds to the sum of $G X$ and $X B$ transition energies. In such nonrephasing 2D FWM, we retrieve the response of $G X B$ systems, whereas exciton complexes without doubly excited states within the excitation bandwidth, such as singly charged QDs, do not contribute.

Figures 5(a) and 5(c) show the measured rephasing and nonrephasing 2D spectra recorded at another position at the sample. In the following, we focus on the two QDs that show up as transitions $G X_{1}$ and $G X_{2}$ on the diagonal of the rephasing spectrum with resonance energies $E_{1}=1359.7 \mathrm{meV}$ and $E_{2}=1358.95 \mathrm{meV}$ - via hyperspectral imaging these are found to be within a $0.5 \mu \mathrm{m}$ vicinity [35] (see Fig. S7 of the Supplemental Material [36] for experimental results regarding the FWM imaging). The peak pattern highlighted by the dashed lines differs from the signatures observed in Fig. 4 in two major respects: First, the spin-orbit coupling of the two circularly polarized excitons within each QD leads to linearly polarized exciton eigenstates, where each QD is described by a four-level system [20]. This causes a splitting of each exciton resonance on the diagonal of the rephasing spectrum into clusters of four peaks, observed in Figs. 5(a) and 5(c). Second, besides the $X_{1} B_{1}$ and $X_{2} B_{2}$ peaks that are redshifted along the FWM axis by the intradot biexciton binding energies $\Delta_{1}=$ $-3.3 \mathrm{meV}$ and $\Delta_{2}=-3.6 \mathrm{meV}$, respectively, we observe two off-diagonal cross peaks labeled $X_{2} X_{1}$ and $X_{1} X_{2}$ at the spectral positions $\left(\omega_{3}=E_{2} ; \omega_{1}=E_{1}\right)$ (upper cross peak) and $\left(\omega_{3}=E_{1} ; \omega_{1}=E_{2}\right)$ (lower cross peak). The appearance of these cross peaks clearly indicates a coherent interdot coupling between the two QDs: The electrostatic Coulomb coupling leads to an energy renormalization of the interdot biexciton $B_{12}$ consisting of one exciton in each QD. The biexciton shift lifts the symmetry of the lower $G X_{1}\left(G X_{2}\right)$ and higher $X_{1} B_{12}$ $\left(X_{2} B_{12}\right)$ transitions, such that the quantum pathways involving these transitions no longer destructively interfere and cross peaks show up [35]. (See Sec. I of the Supplemental Material [36] for theoretical details. A level scheme of the considered QD molecule, including all coupling-induced energy shifts, is shown in Fig. S1.) The electrostatic interaction $\Delta_{12}$ between two excitons located in two different QDs is small compared to the intradot biexciton binding energies $\Delta_{1}$ and $\Delta_{2}$. In fact, the spectrally resolved FWM amplitude [see Fig. S7 of the Supplemental Material [36] for experimental results regarding the spectrally resolved FWM amplitude retrieved from the 2D FWM spectrum shown in Fig. 5(a)] reveals that it is only of the order of $\Delta_{12}=90 \mu \mathrm{eV}$ and it shifts the interdot biexciton towards higher energies, showing up as blueshifted [37] high-energy shoulders of the exciton resonance peaks. This interpretation is supported by calculations [38] of the rephasing and nonrephasing 2D signals depicted in Figs. 5(b) and 5(d) (see Sec. I of the Supplemental Material [36] for details regarding calculations of the FWM response).

In the nonrephasing two-quantum spectrum, the coupling of the two QDs manifests itself in a peak pair labeled $X_{1} B_{12}$ and $X_{2} B_{12}$ at the interaction-shifted two-exciton transition $G B_{12}$ (energy $\omega_{2}=E_{1}+E_{2}+\Delta_{12}=2718.74 \mathrm{meV}$ ) with FWM frequencies $\omega_{3}=E_{1}=1359.7 \mathrm{meV}$ and $\omega_{3}=E_{2}=$ $1358.95 \mathrm{meV}$, respectively. Theoretical calculations (see Sec. I of the Supplemental Material [36] for details regarding calculations of the FWM response) also suggest that exciton transfer processes between the two QDs such as a dipoleinduced (Förster) interaction and Dexter-type coupling via a wave-function overlap are negligible [39]: First, these coupling types are expected to be in the $\mu \mathrm{eV}$ range [35] and therefore difficult to detect considering our spectrometer resolution of $25 \mu \mathrm{eV}$. Second, they would lead to additional peaks for an intradot biexciton in one QD after the first pulse has created a single exciton in the other QD. These peaks are not observed in the spectra, indicating that exciton transfer elements are negligible (see Sec. I C and Fig. S3 of the Supplemental Material [36] for theoretical simulations of the FWM spectra involving different origins of the coherent coupling).

An interesting feature about the observed QD molecule is that, in contrast to the other isolated exciton-biexciton systems, the two coupled QDs show a pronounced finestructure splitting (FSS) of the order of 60 and $140 \mu \mathrm{eV}$, 
respectively. This is around five times higher than the FSS typically present in these QDs [20]. Moreover, the FSS of the other isolated exciton-biexciton systems in our sample (see also Fig. 4) is not visible since the direction of the linear excitation/reference polarization was chosen to be parallel to the anisotropy axis. The observation of such a pronounced FSS only for the resonances associated with the QD molecule therefore suggests that the spatial proximity of the two coupled QDs altered the local symmetry of the confinement, changing the magnitude of the FSS and the polarization of the excitonic transitions.

In summary, we have implemented phase-referenced double quantum 2D FWM spectroscopy of individual quantum systems. By merging it with a single quantum counterpart, we have ascertained coherent couplings between excitons, the structure of (bi)exciton states, and coupling energies in single InAs QDs and in a quantum dot molecule. The optical selection rules of the latter were investigated theoretically.
This methodology is appealing to infer electronic couplings and charge transfer in deterministically defined QD molecules $[40,41]$ and propagative coherence in photonic molecules [42]. By merging it with a recently developed multiwave mixing toolbox [16], it could be also used to visualize and control polaritonic couplings in solid state cavity-quantum electrodynamics [43].

Note added. Recently, we became aware of a report of 2D FWM in rephasing and nonrephasing configurations of individual transitions in interface fluctuation QDs in Ref. [44].

We gratefully acknowledge the financial support by the European Research Council (ERC) Starting Grant PICSEN (Grant No. 306387). J.F.S. and M.R. acknowledge financial support by Deutsche Forschungsgemeinschaft through SFB 787 B1 and GRK 1558 A4. Financial support by the state of Bavaria is acknowledged.
[1] K. Wüthrich, J. Biomol. NMR 27, 13 (2003).

[2] L. M. K. Vandersypen and I. L. Chuang, Rev. Mod. Phys. 76, 1037 (2005).

[3] K. W. Stone, K. Gundogdu, D. B. Turner, X. Li, S. T. Cundiff, and K. A. Nelson, Science 324, 1169 (2009).

[4] D. Turner and K. Nelson, Nature (London) 466, 1089 (2010).

[5] G. Moody, I. A. Akimov, H. Li, R. Singh, D. R. Yakovlev, G. Karczewski, M. Wiater, T. Wojtowicz, M. Bayer, and S. T. Cundiff, Phys. Rev. Lett. 112, 097401 (2014).

[6] K. Hao, L. Xu, P. Nagler, A. Singh, K. Tran, C. K. Dass, C. Schüller, T. Korn, X. Li, and G. Moody, Nano Lett. 16, 5109 (2016).

[7] G. Moody, R. Singh, H. Li, I. A. Akimov, M. Bayer, D. Reuter, A. D. Wieck, A. S. Bracker, D. Gammon, and S. T. Cundiff, Phys. Rev. B 87, 041304 (2013).

[8] G. Moody, R. Singh, H. Li, I. A. Akimov, M. Bayer, D. Reuter, A. D. Wieck, and S. T. Cundiff, Phys. Rev. B 87, 045313 (2013).

[9] E. Cassette, J. C. Dean, and G. D. Scholes, Small 12, 2234 (2016).

[10] H. Wang, K. Ferrio, D. G. Steel, Y. Z. Hu, R. Binder, and S. W. Koch, Phys. Rev. Lett. 71, 1261 (1993).

[11] M. U. Wehner, M. H. Ulm, D. S. Chemla, and M. Wegener, Phys. Rev. Lett. 80, 1992 (1998).

[12] D. S. Chemla and J. Shah, Nature (London) 411, 549 (2001).

[13] J. M. Shacklette and S. T. Cundiff, Phys. Rev. B 66, 045309 (2002).

[14] T. Voss, I. Rückmann, J. Gutowski, V. M. Axt, and T. Kuhn, Phys. Rev. B 73, 115311 (2006).

[15] W. Langbein and B. Patton, Phys. Rev. Lett. 95, 017403 (2005).

[16] F. Fras, Q. Mermillod, G. Nogues, C. Hoarau, C. Schneider, M. Kamp, S. Höfing, W. Langbein, and J. Kasprzak, Nat. Photonics 10, 155 (2016).

[17] Q. Mermillod, T. Jakubczyk, V. Delmonte, A. Delga, E. Peinke, J.-M. Gérard, J. Claudon, and J. Kasprzak, Phys. Rev. Lett. 116, 163903 (2016).

[18] T. Jakubczyk, V. Delmonte, S. Fischbach, D. Wigger, D. E. Reiter, Q. Mermillod, P. Schnauber, A. Kaganskiy, J.-H. Schulze, A. Strittmatter, S. Rodt, W. Langbein, T. Kuhn, S. Reitzenstein, and J. Kasprzak, ACS Photonics 3, 2461 (2016).
[19] S. Maier, P. Gold, A. Forchel, N. Gregersen, J. Mørk, S. Höfling, C. Schneider, and M. Kamp, Opt. Express 22, 8136 (2014).

[20] Q. Mermillod, D. Wigger, V. Delmonte, D. E. Reiter, C. Schneider, M. Kamp, S. Höfling, W. Langbein, T. Kuhn, G. Nogues, and J. Kasprzak, Optica 3, 377 (2016).

[21] J. Kim, S. Mukamel, and G. D. Scholes, Acc. Chem. Res. 42, 1375 (2009).

[22] D. Karaiskaj, A. D. Bristow, L. Yang, X. Dai, R. P. Mirin, S. Mukamel, and S. T. Cundiff, Phys. Rev. Lett. 104, 117401 (2010).

[23] D. B. Turner, P. Wen, D. H. Arias, and K. A. Nelson, Phys. Rev B 84, 165321 (2011).

[24] X. Dai, M. Richter, H. Li, A. D. Bristow, C. Falvo, S. Mukamel, and S. T. Cundiff, Phys. Rev. Lett. 108, 193201 (2012).

[25] B. P. Fingerhut, M. Richter, J.-W. Luo, A. Zunger, and S. Mukamel, Ann. Phys. 525, 31 (2013).

[26] J. Kasprzak, S. Portolan, A. Rastelli, L. Wang, J. D. Plumhof, O. G. Schmidt, and W. Langbein, New J. Phys. 15, 055006 (2013).

[27] W. Langbein, T. Meier, S. Koch, and J. Hvam, J. Opt. Soc. Am. B 18, 1318 (2001).

[28] B. Patton, W. Langbein, U. Woggon, L. Maingault, and H. Mariette, Phys. Rev. B 73, 235354 (2006).

[29] E. J. Mayer, G. O. Smith, V. Heuckeroth, J. Kuhl, K. Bott, A. Schulze, T. Meier, D. Bennhardt, S. W. Koch, P. Thomas, R. Hey, and K. Ploog, Phys. Rev. B 50, 14730(R) (1994).

[30] H. P. Wagner, W. Langbein, and J. M. Hvam, Phys. Rev. B 59, 4584 (1999).

[31] W. Langbein and J. M. Hvam, Phys. Rev. B 61, 1692 (2000).

[32] W. Langbein and J. M. Hvam, Phys. Status Solidi A 190, 167 (2002).

[33] A. D. Bristow, D. Karaiskaj, X. Dai, and S. T. Cundiff, Opt. Express 16, 18017 (2008).

[34] J. Helbing and P. Hamm, J. Opt. Soc. Am. B 28, 171 (2011).

[35] J. Kasprzak, B. Patton, V. Savona, and W. Langbein, Nat. Photonics 5, 57 (2011).

[36] See Supplemental Material at http://link.aps.org/supplemental/ 10.1103/PhysRevB.96.041124 for auxiliary theoretical analysis and experimental results. 
[37] J. Kasprzak and W. Langbein, J. Opt. Soc. Am. B 29, 1766 (2012).

[38] D. Abramavicius, B. Palmieri, D. V. Voronine, F. Sanda, and S. Mukamel, Chem. Rev. 109, 2350 (2009).

[39] J. F. Specht, A. Knorr, and M. Richter, Phys. Rev. B 91, 155313 (2015).

[40] E. A. Stinaff, M. Scheibner, A. S. Bracker, I. V. Ponomarev, V. L. Korenev, M. E. Ware, M. F. Doty, T. L. Reinecke, and D. Gammon, Science 311, 636 (2006).

[41] P.-L. Ardelt, K. Gawarecki, K. Müller, A. M. Waeber, A. Bechtold, K. Oberhofer, J. M. Daniels,
F. Klotz, M. Bichler, T. Kuhn, H. J. Krenner, P. Machnikowski, and J. J. Finley, Phys. Rev. Lett. 116, 077401 (2016).

[42] S. Kapfinger, T. Reichert, S. Lichtmannecker, K. Müller, J. J. Finley, A. Wixforth, M. Kaniber, and H. J. Krenner, Nat. Commun. 6, 8540 (2015).

[43] F. Albert, K. Sivalertporn, J. Kasprzak, M. Strauß, C. Schneider, S. Höfling, M. Kamp, A. Forchel, S. Reitzenstein, E. A. Muljarov, and W. Langbein, Nat. Commun. 4, 1747 (2013).

[44] E. W. Martin and S. T. Cundiff, arXiv:1705.04730. 